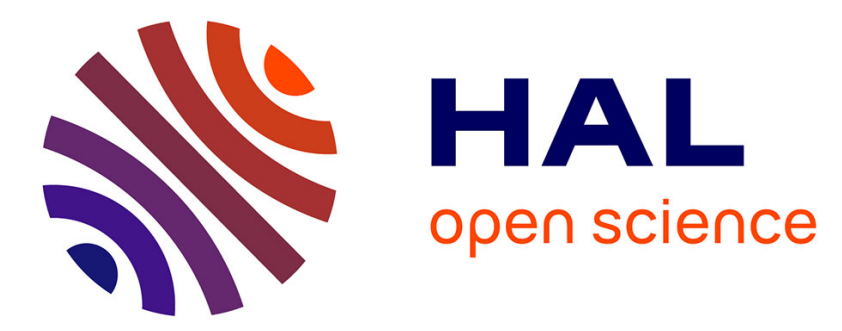

\title{
Investigation of the wall scalar fluctuations effect on pasive scalar turbulent fields at several Prandtl numbers by means of direct numerical simulations \\ Bruno Chaouat, Christophe Peyret
}

\section{- To cite this version: \\ Bruno Chaouat, Christophe Peyret. Investigation of the wall scalar fluctuations effect on pasive scalar turbulent fields at several Prandtl numbers by means of direct numerical simulations. Journal of Heat Transfer, 2019, 141 (12), pp.122002-1 - 122002-9. 10.1115/1.4044882 . hal-02413176}

\author{
HAL Id: hal-02413176 \\ https://hal.science/hal-02413176
}

Submitted on 16 Dec 2019

HAL is a multi-disciplinary open access archive for the deposit and dissemination of scientific research documents, whether they are published or not. The documents may come from teaching and research institutions in France or abroad, or from public or private research centers.
L'archive ouverte pluridisciplinaire HAL, est destinée au dépôt et à la diffusion de documents scientifiques de niveau recherche, publiés ou non, émanant des établissements d'enseignement et de recherche français ou étrangers, des laboratoires publics ou privés. 


\title{
Investigation of the wall scalar fluctuations effect on passive scalar turbulent fields at several Prandtl numbers by means of DNS simulations
}

\author{
Bruno Chaouat *and Christophe Peyret \\ Department of Computational Fluid Dynamics \\ ONERA, Université Paris-Saclay \\ 92322 Châtillon, France
}

\begin{abstract}
We investigate the effect of the wall-scalar fluctuations on passive scalar turbulent fields for a moderate Reynolds number $R_{\tau}=395$ and for several Prandtl numbers ranging from the very low value $P_{r}=0.01$ to the high value $P_{r}=10$ by means of DNS simulations. Several cases of plane channel flows are considered. Case I is a channel flow heated on both walls with a constant imposed heat flux $q_{w}$. We consider for this case two different types of boundary conditions. For the first one, the isoscalar boundary condition $\theta_{w}=0$ is imposed at the wall implying that its fluctuation and therefore its rms scalar fluctuations $\theta_{r m s}=\sqrt{\left\langle\theta^{\prime} \theta^{\prime}\right\rangle}$ is zero at the wall whereas in the second type, $\theta_{w}$ is not prescribed to a fixed value so that it is fluctuating in time at the wall leading to non-zero rms fluctuations. In this latter case, as the heat flux is maintained constant in time at the wall, the fluctuating heat flux $q_{w}^{\prime}$ reduces to zero at the wall. For illustration purpose, in addition to Case I, we also consider Case II which is a plane channel heated only from one wall but cooled from the other one at the same rate taking into account of the free-stream scalar boundary condition at the wall $\theta_{w}^{\prime} \neq 0$ with $q_{w}^{\prime}=0$. The distributions of the mean scalar field, root-mean-square fluctuations, turbulent heat flux, correlation coefficient, turbulent Prandtl number, and
\end{abstract}

\footnotetext{
${ }^{*}$ Corresponding author.
} 
Nusselt number are examined in detail. Moreover, some insights into the flow structure of the scalar fields are provided. As a result of interest, it is found that the mean scalar field $\langle\theta\rangle$ is not affected by the scalar fluctuations at the wall. But owing to the different boundary conditions applied at the wall, significant differences in the evolution of the rms scalar fluctuations $\theta_{\text {rms }}$ are observed in the immediate vicinity of the wall. Surprisingly, the maximum rms intensity remains almost unchanged in the near wall region whatever the type of boundary condition is applied at the wall. In addition, the turbulent heat fluxes that play a major role in heat transfer are found to be independent of the wall scalar fluctuations. This study demonstrates that the impact of the wall scalar fluctuations is appreciable mainly in the near wall region. This outcome must be taken into account when simulating industrial flows with thermal boundary conditions involving different fluid/solid combinations.

\section{Introduction}

Turbulent flows involving the transport of passive scalar are encountered in many engineering applications in industrial plants but also in nature like for instance the pollution dispersal in atmosphere. The passive scalar hypothesis is suitable when the turbulent velocity field governs the scalar field transport while the influence of the scalar field on the velocity field can be neglected as a first approximation. This is the case for instance when the fluid density is not dependent upon temperature, when the scalar field is a mere marker of the fluid particles without mechanical action on them, or when variations of the scalar field are small enough to have negligible action on the fluid motion. Direct numerical simulation (DNS) solving all the turbulence and thermal scales of the problem is the best tool to investigate turbulent scalar fields and it allows also to validate closure models with heat transfer used in Reynolds averaged Navier-Stokes (RANS) modeling [1-4] and large eddy simulation (LES) [5].

Overall, as mentioned by Kasagi et al. [6] and Sommer et al. [7], it is not straightforward to determine the thermal boundary condition on the wall which is in contact with the turbulent flow because of the unsteady heat conduction in the solid due to the unsteadiness of the fluid turbulence. For the solid-fluid conjugate system, the solution at the interface depends on the thermal activity ratio $K=\sqrt{\rho c_{p} \kappa / \rho_{w} c_{p w} \kappa_{w}}$ where $\rho, c_{p}, \kappa$ are the density, specific heat at constant pressure, thermal conductivity, respectively, the subscript $w$ referring to the properties of the wall, and also on the thickness of the wall $[6,8]$. The value of $K$ for combination of air and usual structural materials if very small $10^{-4}, 10^{-3}$ in comparison with liquids where $K$ is of order unity. The isothermal condition is then obtained for $K=0$ corresponding to $\rho_{w} c_{p w} \kappa_{w} \gg \rho c_{p} \kappa$ implying that the wall inertia is high, the wall thick- 
ness is large, and sets the wall temperature fluctuations to zero. In practice, numerical simulations involving heat transfer are often performed assuming the isothermal boundary condition. On the contrary, the isoflux condition is obtained for $K$ going to infinity with $\rho_{w} c_{p w} \kappa_{w} \ll \rho c_{p} \kappa$, suggesting consequently that the wall inertia is small, the wall thickness is infinitesimal, and then that the wall temperature fluctuations are not zero. This outcome is confirmed by the experimental study of the wall temperature fluctuations conducted by Mosyak et al. [9] indicating that the fluctuating temperatures are of higher intensity for the isoflux condition than for the isothermal condition. As mentioned by Matsubara et al. [10], the isoflux condition should be considered from an academic point of view to the near wall side behavior in heating condition.

As the problem of thermal boundary conditions at the wall is necessarily encountered in heat transfer simulations, and that the temperature fluctuations at the wall may lead to thermal fatigues failure of solid structures, it is of importance to investigate the impact of the scalar fluctuations at the wall on passive scalar turbulent fields, even if the simulations are performed on the fluid side only. The simple way to do that with the highest degree of accuracy possible is to perform direct numerical simulations (DNS) solving all the turbulence and thermal scales in the flow. We shall consider the plane channel flow heated on both walls with a constant imposed heat flux (Case I) as shown in Fig. 1 with firstly zero scalar fluctuations at the wall and secondly with free scalar fluctuations at the wall. The case with zero temperature fluctuations at the wall has been studied in the past by several authors at different Reynolds and Prandtl numbers but so far, not for the one associated with wall fluctuations which would deserve particular interest. Among these authors, one can mention Kim and Moin [11] for $R_{\tau}=180$ with $P_{r}=0.1,0.71,2$; Kasagi et al. [12] for $R_{\tau}=180$ with $P_{r}=0.1,0.71,2$; Kasagi and Shikazono [13] for $R_{\tau}=150$ with $P_{r}=0.025,0.71$; Kawamura et al. [14] for $R_{\tau}=180$ with $P_{r}=0.025,0.05,0.2,0.4,0.71,1.0,1.5$, 5; Kawamura et al. [15] for $R_{\tau}=150,180,395$ with $P_{r}=0.025,0.2,0.71$; Na and Hanratty [16] for $R_{\tau}=150$ with $P_{r}=1$, 3, 10; Abe et al. [17] for $R_{\tau}=180,395,640,1020$ with $P_{r}=0.025,0.71$; Kozuka et al. [18] for $R_{\tau}=180$, 395 with $P_{r}=0.71,1,2,7,10$. Physically, the Prandtl numbers chosen are representative of heat transfer in liquid metals such as mercury or liquid sodium $\left(P_{r} \approx 0.015\right)$, gases $\left(P_{r} \approx 1\right)$, water $\left(P_{r} \approx 5-7\right)$, and seawater $\left(P_{r} \approx 13\right.$ at $0^{\circ} \mathrm{C}$ ) depending on the temperature.

As pointed out above, the case of a fully developed thermal flow subjected to free scalar fluctuations at the wall was not reported by previous DNS studies in the literature. We can only mention another case concerned with open channel flows performed by Tiselj et al. [8] for $R_{\tau}=171$ with $P_{r}=1.54$ and by Lu and Hetsroni [19] 


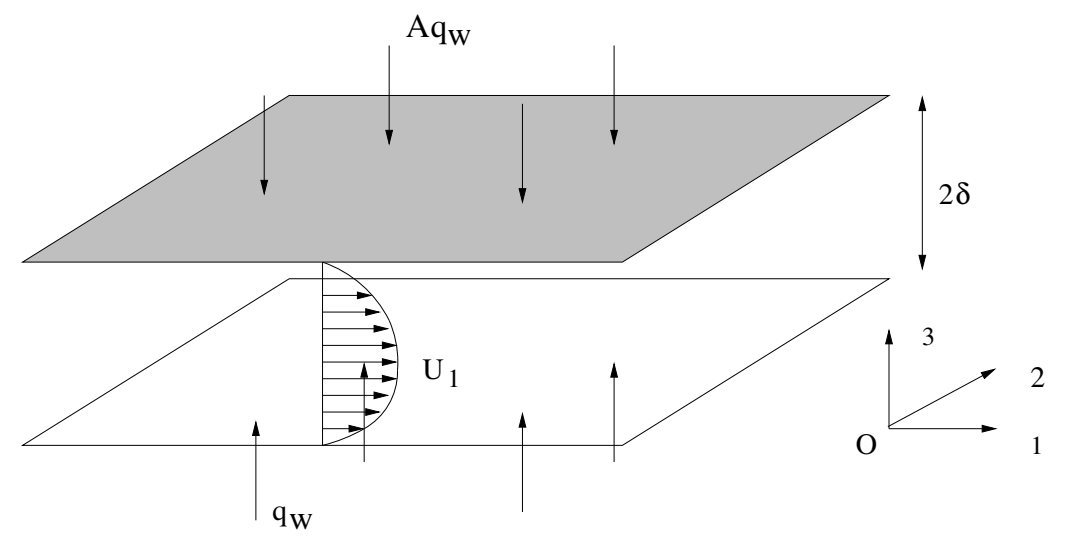

Fig. 1. Setup of the numerical channel flow simulations with various thermal boundary conditions. Both walls are heated $(A=1)$; One wall is heated but the other one is cooled at the same rate $(A=-1)$.

for $R_{\tau}=180$ with $P_{r}=0.71$. Unfortunately, no previous studies have addressed the Prandtl number dependence since only moderate Prandtl numbers were selected. If these simulations have however provided some trends in the behavior of the wall fluctuation effect in the case of an open channel flow at such moderate Prandtl numbers, there is a need to perform the fully turbulent channel flow bounded by lower and upper walls, aiming to calibrate the effect of the wall temperature fluctuations on the scalar field at different Prandtl numbers ranging from low to high values. This case is generic to many industrial flows. This issue concerning wall fluctuations at the wall is thus of primary importance in CFD involving heat transfer.

In the present work, we will perform DNS of the channel flow at the Reynolds number $R_{\tau}=395$ and at the Prandtl numbers $P_{r}=0.01,0.1,1,10$ corresponding to Case I with the two different wall boundary conditions including zero wall fluctuations and non-zero free-stream wall fluctuations $\theta_{w}^{\prime}$, respectively. A special attention is devoted to the impact of the instantaneous fluctuations $\theta_{w}^{\prime}$ on the scalar turbulent fields. We will provide elements of comparison between these two scalar fields for each Prandtl number. In particular, we will focus on the mean scalar variable and rms fluctuations but also on the turbulent heat fluxes. Furthermore, we will examine the validity of the turbulent flux modeling based on the well known gradient law hypothesis. The structure of the scalar fields will be then investigated for each Prandtl number. For illustration purpose, in addition to Case I, we will consider also Case II corresponding to the plane channel heated only from one wall but cooled from the other one (see Fig. 1) at the same rate accounting for free scalar fluctuations at the wall. In this work, the passive scalar can be interpreted as temperature but more generally as any passive contaminant associated with concentration or mass transfer. 


\section{Equations, boundary conditions and numerical procedure}

\subsection{Equations}

The Navier-Stokes equations of the velocity field expressing the conservation of mass and momentum are

$$
\frac{\partial u_{j}^{+}}{\partial x_{j}^{*}}=0
$$

and

$$
\frac{\partial u_{i}^{+}}{\partial t^{*}}+\frac{\partial}{\partial x_{j}^{*}}\left(u_{i}^{+} u_{j}^{+}\right)=-\frac{\partial p^{+}}{\partial x_{i}^{*}}+\frac{1}{R_{\tau}} \frac{\partial^{2} u_{i}^{+}}{\partial x_{j}^{*} \partial x_{j}^{*}}+G_{i}
$$

where in these equations, $u_{i}$ denotes the velocity and $p$ is the pressure. The time $t$, coordinate $x_{i}$ and flow variables are normalized using the channel half width $\delta$, the friction velocity $u_{\tau}$ and the kinematic viscosity $v$ such as $t^{*}=t u_{\tau} / \delta, x_{i}^{*}=x_{i} / \delta, u_{i}^{+}=u_{i} / u_{\tau}, p^{+}=p / \rho u_{\tau}^{2}$. The quantity $G$ included in this equation denotes the mean pressure gradient term necessary to balance friction at the upper and lower walls allowing to get periodic condition between the inlet and outlet sections of the channel. In dimensionless form, $G_{1}$ takes on the value unity. The transport equation for the passive scalar $\theta$ reads

$$
\frac{\partial \theta^{+}}{\partial t^{*}}+\frac{\partial}{\partial x_{j}^{*}}\left(\theta^{+} u_{j}^{+}\right)=\frac{1}{R_{\tau} P_{r}} \frac{\partial^{2} \theta^{+}}{\partial x_{j}^{*} \partial x_{j}^{*}}+Q
$$

where in this equation, the velocity $u_{i}$ is given by Eq. (2), and $Q$ denotes the source term. Its physical meaning corresponds to the mean temperature gradient necessary to balance wall heat fluxes. It can be easily demonstrated by making a function change in the temperature with a linear function. The variable $\theta$ is normalized by the wall characteristic transfer temperature defined as $\theta_{\tau}=q_{w} /\left(\rho c_{p} u_{\tau}\right)$. The heat flux is given by $q_{w}=-\kappa\left(\partial \theta / \partial x_{3}\right)_{w}$ where $\kappa=\rho c_{p} \vee / P_{r}$ stands for the thermal conductivity and the thermal diffusivity is given by $\sigma=\kappa /\left(\rho c_{p}\right)=\mathrm{v} / P_{r}$. Two different boundary conditions are used for the scalar field according to the parameter A (see Fig. 1). In Case I, $A=1$, both walls are heated and $Q=-u_{1}^{+} / U_{b}^{+}$allows to ensure zero gradient of the mean passive scalar $\partial\langle\theta\rangle / \partial x_{1}=0$, whereas in Case II, $A=-1, Q=0$, i.e, an internal heat source is not required to get a fully 
developed thermally flow. Note that the averaged transport equation for the passive scalar is given by

$$
\frac{\partial\left\langle\theta^{+}\right\rangle}{\partial t^{*}}+\frac{\partial}{\partial x_{j}^{*}}\left(\left\langle u_{j}^{+}\right\rangle\left\langle\theta^{+}\right\rangle\right)=\frac{1}{R_{\tau} P_{r}} \frac{\partial^{2}\left\langle\theta^{+}\right\rangle}{\partial x_{j}^{*} \partial x_{j}^{*}}-\frac{\partial}{\partial x_{j}^{*}}\left\langle u_{j}^{++} \theta^{\prime+}\right\rangle+Q
$$

where in this equation, the turbulent heat fluxes $\left\langle u_{j}^{\prime+} \theta^{\prime+}\right\rangle$ deserves a particular attention in heat transfer modeling in both RANS and LES methodologies [2,4,20].

\subsection{Boundary conditions}

In both cases, the boundary conditions at the lower and upper walls for $x_{3}=0$ and $2 \delta$ are no slip velocity conditions $u_{i}^{+}=0$. A constant heat flux corresponding to an isoflux boundary condition given by $q_{w}=-\kappa\left(\partial \theta / \partial x_{3}\right)_{w}$ is applied at the wall. We consider then two limiting cases recalled hereafter. Firstly, the case where $K \rightarrow 0$ corresponding to the isoscalar boundary condition $\theta_{w}=0$ implying that the fluctuation $\theta_{w}^{\prime}=0$ and leading to zero $r m s$ scalar fluctuations $\theta_{r m s, w}=0$ [18]. Secondly, the case where $K \rightarrow \infty$ corresponding to the free-stream scalar condition, i.e, $\theta_{w}$ is not prescribed to a constant value implying that $\theta_{w}^{\prime} \neq 0$ and leading to non zero $r m s$ fluctuations $\theta_{r m s, w} \neq 0$ [6]. As the heat flux is maintained constant in time at the wall, the fluctuating heat flux $q_{w}^{\prime}$ reduces to zero at the wall, $q_{w}^{\prime}=-\kappa\left(\partial \theta^{\prime} / \partial x_{3}\right)_{w}=0$, so that the derivative of the scalar fluctuation along the normal direction $x_{3}$ verifies $\left(\partial \theta^{\prime} / \partial x_{3}\right)_{w}=0$ leading to $\left(\partial \theta_{r m s} / \partial x_{3}\right)_{w}=0$. This means that the variance of the passive scalar is almost constant along the normal to the wall in the immediate vicinity of the wall.

\subsection{Numerical procedure}

The dimension of the channel in the streamwise, spanwise and normal directions along the axes $x_{1}, x_{2}, x_{3}$ are $L_{1}=6.4 \delta, L_{2}=3.2 \delta$ and $L_{3}=2 \delta$. The number of grid points is determined in order to solve both the Kolmogorov scale given by $\eta_{\kappa}=\left(v^{3} / \varepsilon\right)^{1 / 4}$ and the Batchelor length-scale $\eta_{\theta}$ [21-23] which approaches $\eta_{\kappa}$ when $P_{r}$ is of order of unity, $\eta_{\theta}=\left(\sigma^{3} / \varepsilon\right)^{1 / 4}=\eta_{\kappa} / P_{r}^{3 / 4}$ at small Prandtl numbers and $\eta_{\theta}=\left(v \sigma^{2} / \varepsilon\right)^{1 / 4}=\eta_{\kappa} / P_{r}^{1 / 2}$ at large Prandtl numbers. Thus, the resolution of the grid for a direct numerical simulation of scalar turbulence is loose at low Prandtl number but on the contrary, it will be very fine at large Prandtl number according to the power law of the Prandtl number. For instance, at $P_{r}=0.01, \eta_{\theta} \approx 31.6 \eta_{\kappa}$, at $P_{r}=0.1, \eta_{\theta} \approx 5.62 \eta_{\kappa}$, at $P_{r}=1, \eta_{\theta} \approx \eta_{\kappa}$ but at $P_{r}=10, \eta_{\theta} \approx 0.316 \eta_{\kappa}$. So that the number of grid points behaves likes $N \propto R_{t}^{9 / 4} P_{r}^{3 / 2}$ at large Prandtl numbers where $R_{t}=k^{2} /(v \varepsilon)$ is the turbulent Reynolds number. For the Reynolds number and Prandtl number 


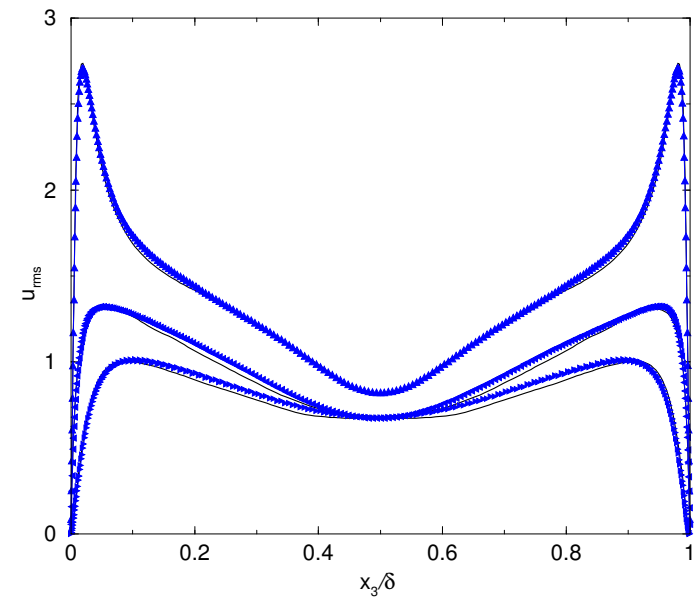

Fig. 2. Turbulence intensities $u_{i r m s}^{+}=\sqrt{\left\langle u_{i}^{\prime+} u_{i}^{\prime+}\right\rangle}$ versus the wall coordinate. Present DNS performed on the mesh $M_{1}: \mathbf{\Lambda}, \mathrm{i}=1 ; \boldsymbol{4}, \mathrm{i}=2$; >, i=3. DNS (Moser et al. 1999), $-R_{\tau}=395$.

values studied here, the grid numbers vary from the mesh $M_{1}$ with resolution $256 \times 128 \times 256$ for $P_{r}=0.01$ and 0.1 to $M_{2}$ with resolution $512 \times 256 \times 256$ for $P_{r}=1$ and $M_{3}$ with resolution $1024 \times 512 \times 512$ for $P_{r}=10$. For all these meshes, the spacings are given by $\Delta_{i}^{+}=R_{\tau} L_{i} / N_{i} \delta$ leading to $\Delta_{1}^{+}=\Delta_{2}^{+} \approx 10$ for $M_{1}, \Delta_{1}^{+}=\Delta_{2}^{+} \approx 5$ for $M_{2}$ and $\Delta_{1}^{+}=\Delta_{2}^{+} \approx 2.5$ for $M_{3}$. Note that in this latter case, the grid spacing in the normal direction to the wall is very small $\Delta_{3}^{+}<2$. The equations are integrated in time using an explicit Runge-Kutta scheme of fourth order accuracy in time and solved in space by means of a centered scheme of fourth order accuracy in space. The CFD code developed by Chaouat [24] is based on the finite volume technique and is optimized with message passing interface (MPI).

\section{Turbulent channel flow with isotherm walls}

First at all, we compare the numerical simulation of the turbulent channel flow performed on the mesh $M_{1}$ with the DNS of Moser et al. [25] at the Reynolds number $R_{\tau}=395$ to confirm that the flow field is well resolved on the velocity grid. For that purpose, Fig. 2 shows the turbulence intensities of the fluctuating velocities. As expected, an excellent agreement is observed between the present DNS and the reference data. In particular, the peaks of turbulence are perfectly reproduced in the near wall-region. This result indicates that the mesh $M_{1}$ and therefore the meshes $M_{2}$ and $M_{3}$ which are of higher grid resolution than $M_{1}$ are sufficiently refined to successfully reproduce the rms fluctuations of turbulence. This short section concludes that the numerical code returns accurate results. 


\section{Turbulent channel flow heated on both walls with and without scalar fluctuations at the wall (Case I)}

\subsection{Statistics of passive scalars}

The transformed variable $\Theta^{+}=\theta_{w}^{+}-\theta^{+}$is considered to analyze the present results. In this Case I, the mean statistical quantities are symmetric about the centerline of the channel, the gradients of the scalar field including the mean and rms fluctuations reduce to zero at the center of the channel.

\subsubsection{Mean scalar field}

Figure 3 describes the profile of the mean scalar variable $\Theta^{+}$versus the logarithmic wall distance for different Prandtl numbers returned by the simulations with and without the scalar fluctuations condition at the wall. As known, when the Prandtl number increases from 0.01 to 10 , the conduction region penetrates less deeply into the core region of the channel leading to an increase of the scalar variable, the logarithmic region is then extended. At a first sight, it is found that there are no discernable differences between the mean profiles associated with the two different boundary conditions in the wall region. The curves overlap each other and cannot be distinguished from the symbols. Consequently, these results demonstrate that the fluctuations at the walls have no practical effect on the mean scalar profile.

\subsubsection{Root mean square of the scalar variance}

Figure 4 displays the rms of the scalar variance for each Prandtl number. As the Prandtl number increases, the rms fluctuation intensities also increase, and the peak value location moves close to the wall. As expected, the profiles of the rms fluctuations differ from each other in the wall region according to the wall boundary condition. Indeed, it is found that the impact of the wall fluctuations on the scalar turbulent field is appreciable especially within the immediate vicinity of the wall. The rms fluctuation intensities associated with the isoscalar condition return to zero whereas those associated with the isoflux condition $q_{w}^{\prime}=0$ are non-zero at the wall. In this latter case $\left(q_{w}^{\prime}=0\right)$, it can be seen that the rms of the scalar fluctuations is almost constant in the vicinity of the wall, i.e, its gradient in the normal direction to the wall is zero, $\partial \theta_{r m s} / \partial x_{3}=0$. Surprisingly, the maximum of the $r m s$ intensity remains almost unchanged in the near wall region whatever the wall boundary condition considered, even if the level of the rms is very high at the wall. The different curves which were departing from one another at the wall, tend to merge after the location of the peak of the scalar variance, confirming that the wall effect seems to be fading rapidly as the wall distance increases. Moreover, when the Prandtl number increases from 0.01 to 10 , the peak of the scalar variance moves toward the wall so that the effect of the wall scalar fluctuations on the 


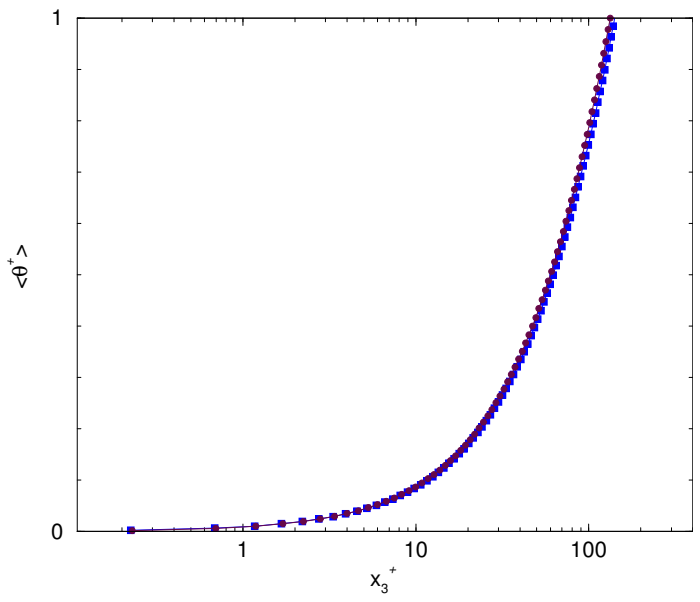

(a)

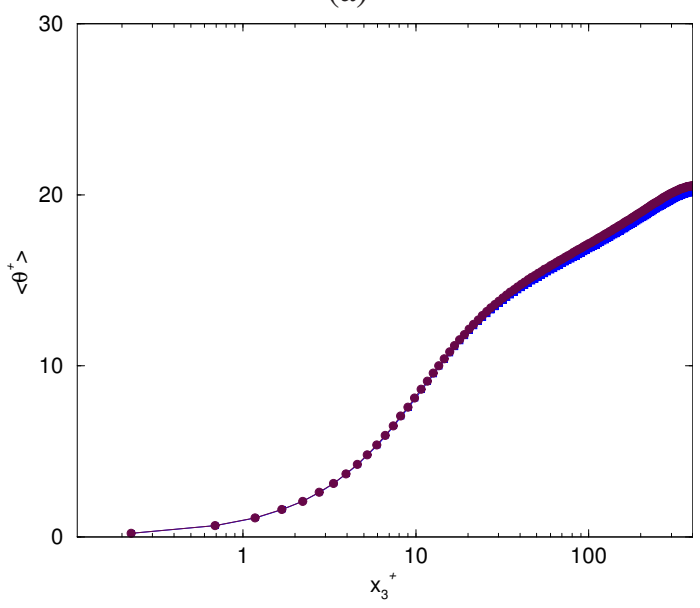

(c)

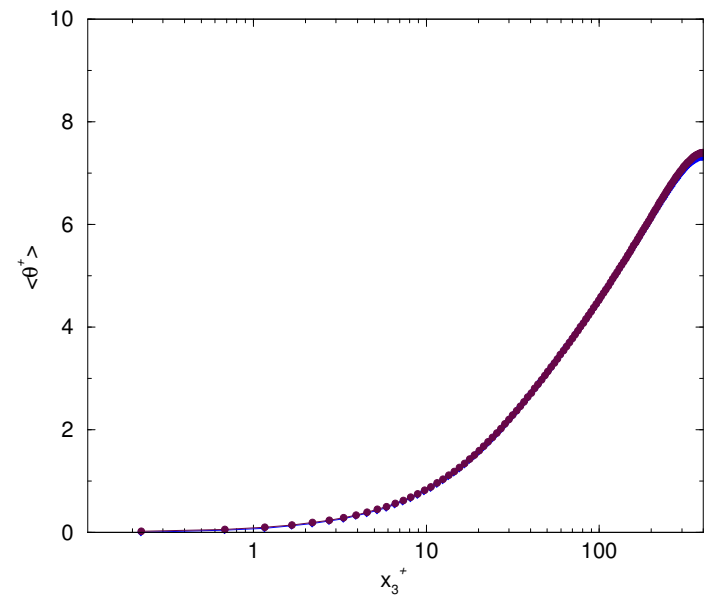

(b)

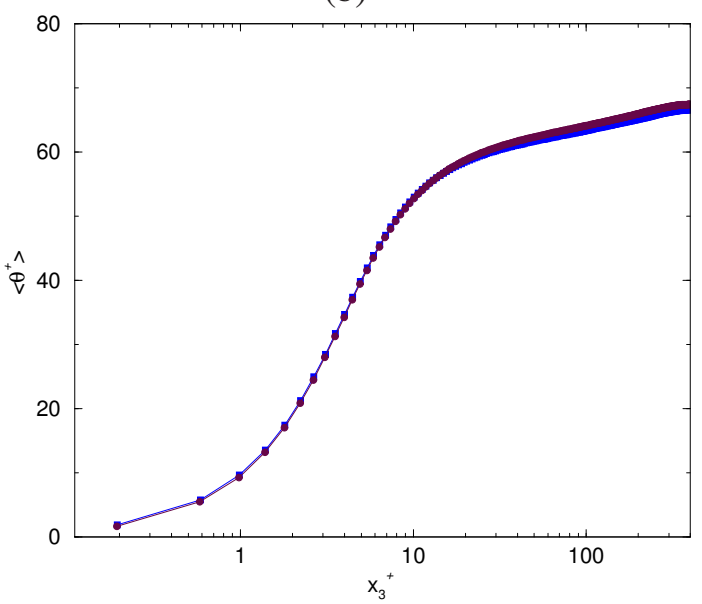

(d)

Fig. 3. Mean scalar field $\left\langle\theta^{+}\right\rangle$at several Prandtl numbers in logarithmic coordinate versus the wall unit distance. $\theta_{r m s, w}=0, \bullet \cdot q_{w}^{\prime}=0$, Ш. (a) $P_{r}=0.01$; (b) $P_{r}=0.1$; (c) $P_{r}=1$; (d) $P_{r}=10 ; R_{\tau}=395$.

scalar field appears more marked at low Prandtl numbers than at high Prandtl numbers. In particular, at $P_{r}=0.01$, the $r m s$ profiles strongly differ between each other in the boundary layer region ranging from $x_{3}^{+}=0$ to roughly 180 whereas at $P_{r}=10$, the difference is only visible in the immediate vicinity of the wall from $x_{3}^{+}=0$ to the wall unit distance 6 . This outcome also confirms that the grids must be sufficiently refined in the near wall region to accurately reproduce the thermal boundary layer, especially at high Prandtl numbers since the length-scale $\eta_{\theta}$ dramatically decreases according to the Batchelor law $\eta_{\theta}=\eta_{\kappa} / P_{r}^{1 / 2}$.

\subsubsection{Turbulent heat fluxes}

The turbulent heat flux $q_{i}^{+}=-\left\langle u_{i}^{\prime+} \theta^{\prime+}\right\rangle$ appearing in Eq. (4) plays a pivotal role in the determination of the heat transfer of turbulent fluid. The direct numerical simulations performed here for several Prandtl numbers 


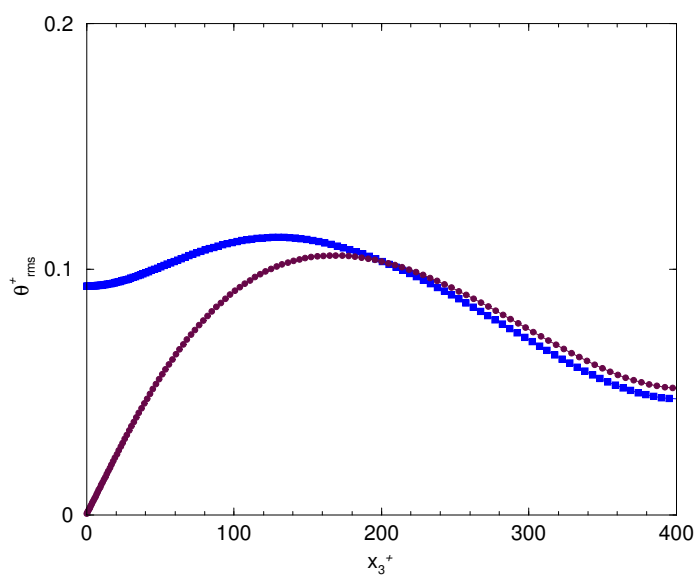

(a)

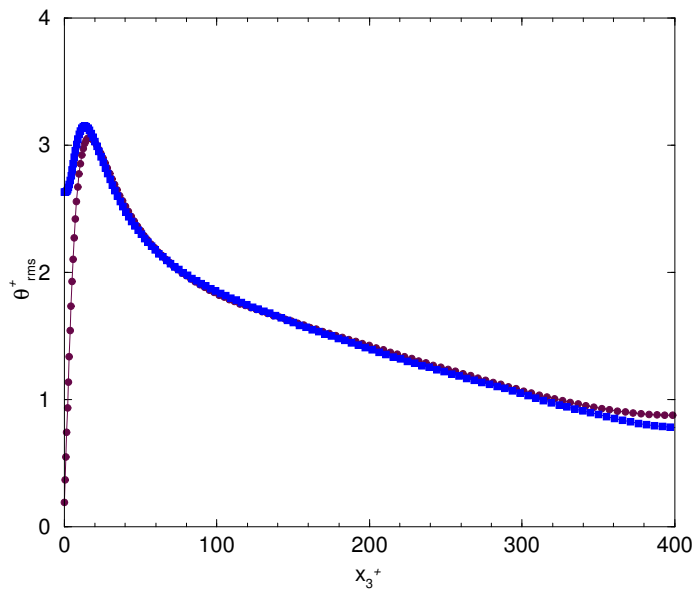

(c)

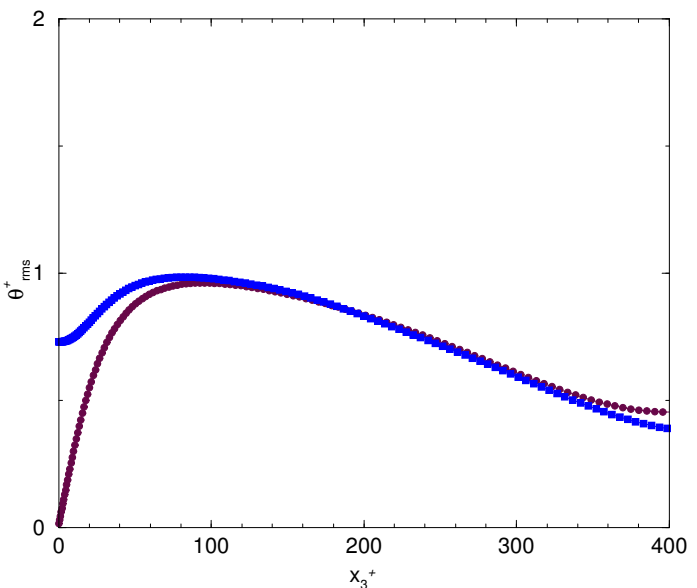

(b)

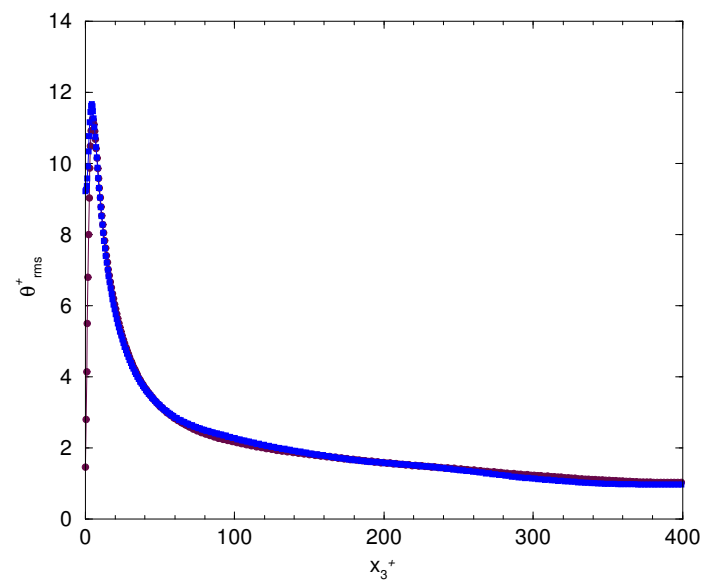

(d)

Fig. 4. Root mean square of the scalar variance $\theta_{r m s}^{+}=\sqrt{\left\langle\theta^{\prime+} \theta^{\prime+}\right\rangle}$ at several Prandtl numbers versus the wall unit distance. $\theta_{r m s, w}=$

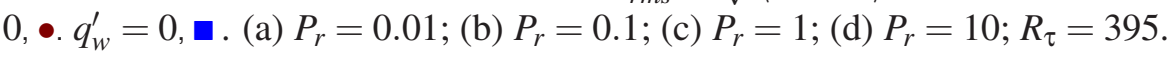

provide reference data for all quantities. In particular, the computation of $q_{i}$ from DNS is very useful for calibrating RANS and LES models. Figures 5 and 6 display the profile of the turbulent heat fluxes in the wall-normal direction $q_{3}^{+}=-\left\langle u_{3}^{\prime+} \theta^{\prime+}\right\rangle$ and in the streamwise direction $q_{1}^{+}=-\left\langle u_{1}^{\prime+} \theta^{\prime+}\right\rangle$, respectively, versus the dimensionless wall coordinate for all Prandtl numbers. By integrating Eq. (4), it is simple matter to show that the normal turbulent heat flux satisfies the following relation

$$
\left\langle u_{3}^{\prime+} \theta^{\prime+}\right\rangle=1-\frac{1}{P_{r}} \frac{\partial\left\langle\Theta^{+}\right\rangle}{\partial x_{3}^{+}}-\frac{1}{R_{\tau} U_{b}^{+}} \int_{0}^{x_{3}^{+}}\left\langle u_{1}^{+}\right\rangle d x_{3}^{+}
$$


where $x_{i}^{+}=x_{i} u_{\tau} / \mathrm{v}$ and $U_{b}$ denotes the bulk velocity. The total turbulent heat flux is defined as the sum of the conductive and turbulent heat fluxes as

$$
q_{3 t}^{+}=\frac{1}{P_{r}} \frac{\partial\left\langle\Theta^{+}\right\rangle}{\partial x_{3}^{+}}-\left\langle u_{3}^{\prime+} \theta^{\prime+}\right\rangle
$$

At low Prandtl numbers, the conductive heat fluxes largely exceed the turbulent heat fluxes due to high molecular fluid conductivity. Although the normal heat flux remains lower than unity according to Eq. (5), the spanwise heat flux is however much greater than unity. The turbulent heat fluxes highly increase in the immediate vicinity of the wall with the increase of the Prandtl number and then gradually decrease when moving to the central region of the channel. The correlation $\left\langle u_{3}^{\prime} \theta^{\prime}\right\rangle$ changes of sign when passing from the lower wall to the upper wall $\left(u_{3}^{\prime}>0\right.$, $\theta^{\prime}>0$ for $x_{3}<\delta$ and $u_{3}^{\prime}<0, \theta^{\prime}>0$ for $x_{3}>\delta$ ) leading to an anti-symmetric profile. Consequently, the normal turbulent heat flux $q_{3}=-\left\langle u_{3}^{\prime+} \theta^{\prime+}\right\rangle$ returns to zero in the centerline of the channel. A different situation occurs for the streamwise turbulent heat flux. The correlation $\left\langle u_{1}^{\prime} \theta^{\prime}\right\rangle$ remains of the same sign when passing from the lower wall to the upper wall $\left(u_{1}^{\prime}>0, \theta^{\prime}>0\right.$ for $\left.x_{3}<2 \delta\right)$ leading to a symmetric profile. As a result, it is found that the profiles of the turbulent heat fluxes are roughly the same whatever the boundary condition applied at the wall. The turbulent heat fluxes accounting for the wall fluctuations are just a bit larger in term of intensity. This outcome means that the wall fluctuations have a very slight effect on the turbulent heat fluxes that play a crucial role in thermal transfer of turbulent flows.

\subsubsection{Correlation coefficient}

The correlation coefficient between the passive scalar and the velocity is defined by

$$
R_{i \theta}=\frac{\left\langle u_{i}^{\prime} \theta^{\prime}\right\rangle}{\sqrt{\left\langle u_{i}^{\prime} u_{i}^{\prime}\right\rangle} \sqrt{\left\langle\theta^{\prime} \theta^{\prime}\right\rangle}}
$$

Figures 7 and 8 display the profiles of the correlation coefficients $R_{3 \theta}$ and $R_{1 \theta}$, respectively, versus the wall unit distance. As expected, the profile of the correlation coefficient $R_{3 \theta}$ is anti-symmetric with respect to the centerline of the channel so that $R_{3 \theta}$ returns to zero in the central region of the channel whereas the profile of $R_{1 \theta}$ is symmetric leading to zero gradient in the centerline. As the velocity and scalar fluctuations are of the same sign for $R_{1 \theta}$ but 


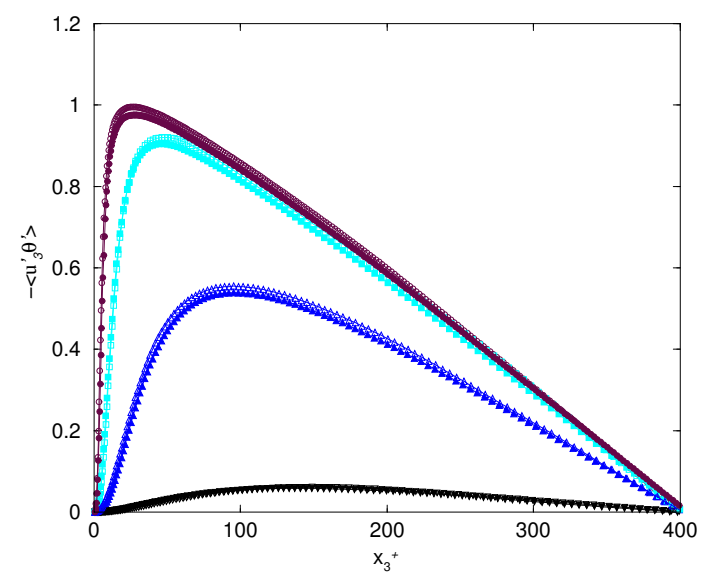

Fig. 5. Normal turbulent heat flux $q_{3}=-\left\langle u_{3}^{\prime+} \theta^{\prime+}\right\rangle$ versus the wall unit distance. $\theta_{r m s, w}=0: P_{r}=0.01, \mathbf{\nabla} ; P_{r}=0.1, \mathbf{\Lambda} . P_{r}=1$, 匹. $P_{r}=10, \bullet . q_{w}^{\prime}=0: P_{r}=0.01, \nabla ; P_{r}=0.1, \Delta . P_{r}=1, \square . P_{r}=10, \circ . R_{\tau}=395$.

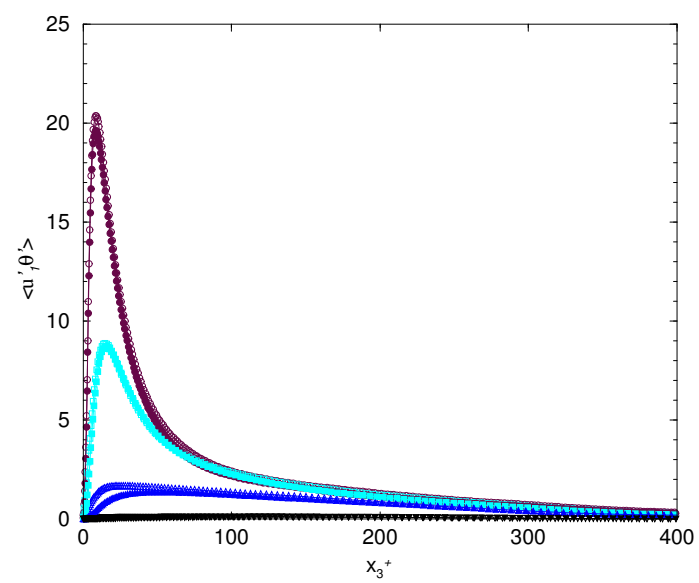

Fig. 6. Streamwise turbulent heat flux $\left|q_{1}\right|=\left\langle u_{1}^{\prime+} \theta^{\prime+}\right\rangle$ versus the wall unit distance. $\theta_{r m s, w}=0: P_{r}=0.01, \mathbf{\nabla} ; P_{r}=0.1$, $P_{r}=1, \square . P_{r}=10, \bullet q_{w}^{\prime}=0: P_{r}=0.01, \nabla ; P_{r}=0.1, \Delta . P_{r}=1, \square . P_{r}=10, \circ . R_{\tau}=395$.

change of sign for $R_{3 \theta}$, the correlation coefficient $R_{1 \theta}$ is of higher magnitude than $R_{3 \theta}$. It appears also that $R_{1 \theta}$ reaches its maximum value in the wall region at $P_{r}=1$ suggesting the presence of streaks in the flowfield. The similarity between the velocity and scalar fields becomes weaker as the Prandtl number departs from unity, both fields are then governed by different turbulent mechanisms. On overall, it is found that $R_{3 \theta}$ accounting for the scalar fluctuations at the wall is of higher magnitude than for the case with no fluctuation at the wall. The reason is due to the correlation $\left\langle\theta^{\prime} \theta^{\prime}\right\rangle$ that is more affected by the wall fluctuations than the correlation $\left\langle u_{3}^{\prime} \theta^{\prime}\right\rangle$ as observed in the preceding figures 4 and 5 to 6 . This effect is however less marked as the Prandtl number increases. In particular, the curves corresponding to the same Prandtl number exhibit large departures between each other at $P_{r}=0.01$ but are however almost identical at $P_{r}=10$. 


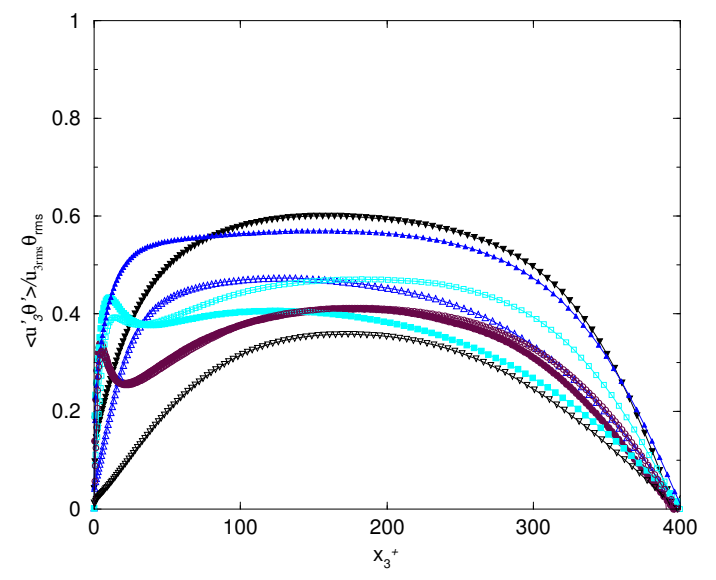

Fig. 7. Correlation coefficient of the wall-normal turbulent heat flux $R_{3 \theta}$ versus the wall unit distance. $\theta_{r m s, w}=0: P_{r}=0.01, \boldsymbol{\nabla}$; $P_{r}=0.1, \boldsymbol{\Delta} . P_{r}=1$, ․ $P_{r}=10, \bullet . q_{w}^{\prime}=0: P_{r}=0.01, \nabla ; P_{r}=0.1, \Delta . P_{r}=1, \square . P_{r}=10, \circ . R_{\tau}=395$.

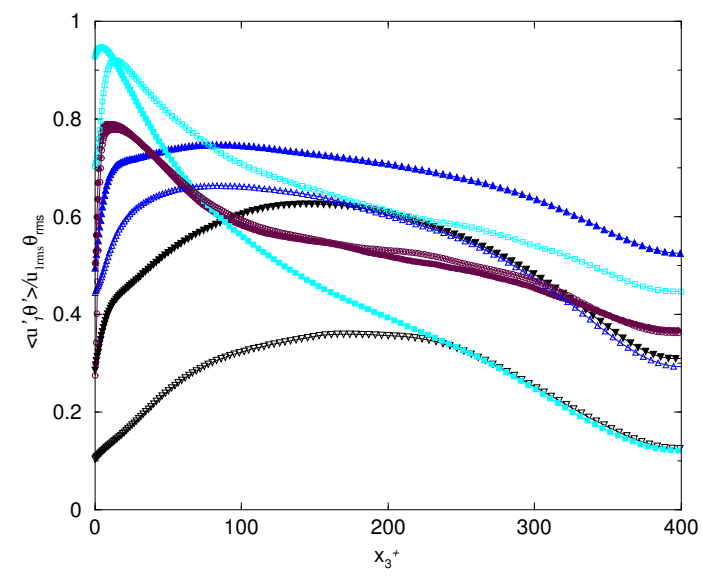

Fig. 8. Correlation coefficient of the streamwise turbulent heat flux $R_{1 \theta}$ versus the wall unit distance. $\theta_{r m s, w}=0: P_{r}=0.01, \mathbf{\nabla}$; $P_{r}=0.1, \boldsymbol{\Delta} . P_{r}=1, \square . P_{r}=10, \bullet . q_{w}^{\prime}=0: P_{r}=0.01, \nabla ; P_{r}=0.1, \Delta . P_{r}=1, \square . P_{r}=10, \circ . R_{\tau}=395$.

\subsubsection{Turbulent Prandtl number}

We now examine the validity of the gradient law hypothesis of the turbulent heat flux modeling

$$
\left\langle\theta^{\prime} u_{3}^{\prime}\right\rangle=-\frac{v_{t}}{P r_{t}} \frac{\partial\langle\theta\rangle}{\partial x_{3}}
$$


where $P_{r t}$ denotes the turbulent Prandtl number and $v_{t}$ stands for the turbulent eddy viscosity. The Prandtl number is defined itself as the ratio of the turbulent eddy viscosity $v_{t}$ to the turbulent eddy diffusivity $\sigma_{t}$

$$
P r_{t}=\frac{\left\langle u_{1}^{\prime} u_{3}^{\prime}\right\rangle \partial\langle\theta\rangle / \partial x_{3}}{\left\langle\theta^{\prime} u_{3}^{\prime}\right\rangle \partial\left\langle u_{1}\right\rangle / \partial x_{3}}
$$

Figure 9 shows the profile of the turbulent Prandtl number versus the wall unit distance for all simulations. In the Prandtl numbers range $P_{r}=0.1,1$ to 10 , the turbulent Prandtl number reaches an asymptotic behavior independent of the molecular Prandtl number. It is close to unity except however in the near wall region where a very small variation of a few percent is observed. This result validates the hypothesis of a constant turbulent Prandtl number at moderate and high molecular Prandtl numbers. But at $P_{r}=0.01$, the turbulent Prandtl number varies strongly with respect to the wall coordinate. The shape of this profile is due to the strong conductive effects acting in low Prandtl number flows. Indeed, the scalar fluctuations are dissipated faster as the Prandtl number decreases

implying that the correlation $\left\langle u_{3}^{\prime} \theta^{\prime}\right\rangle$ appearing in the denominator of Eq. (9) is lower. As a result of interest, it is found that the profiles associated with and without the scalar fluctuations at the wall are somewhat different in the wall region, especially at low Prandtl numbers. The mean velocity and scalar gradient terms appearing in Eq. (9) are almost identical in presence or not of the fluctuations at the wall, so that this difference should be attributed to the correlation $\left\langle u_{3}^{\prime} \theta^{\prime}\right\rangle$ that is slightly modified by the wall fluctuations. Case I is generic to many industrial applications involving heat transfer and reveals that the assumption of a constant turbulent Prandtl number still holds at moderate and high molecular Prandtl numbers regardless of the thermal boundary condition applied at the wall.

\subsubsection{Nusselt number}

Figure 10 exhibits the profile of the mean Nusselt number defined as

$$
N u=\frac{2 \delta h}{\kappa}=\frac{2 \delta q_{w}}{\kappa\left(\theta_{w}-\theta_{b}\right)}=\frac{2 R_{\tau} P_{r}}{\theta_{w}^{+}-\theta_{b}^{+}}
$$

versus the Prandtl number where in this equation, $\theta_{b}$ denotes the bulk scalar variable and $h$ stands for the heat transfer coefficient defined as $h=q_{w} /\left(\theta_{w}-\theta_{b}\right)$. As Fig. 3 has shown that the mean scalar profile remains the 


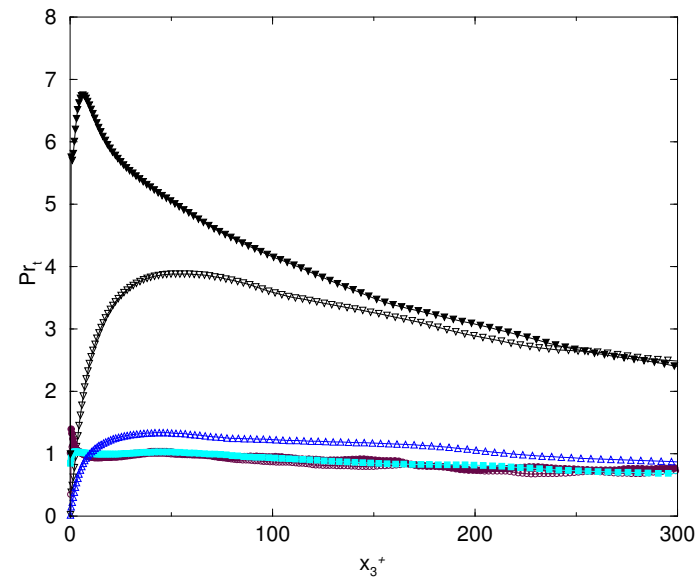

Fig. 9. Profiles of the turbulent Prandtl number versus the wall unit distance. $\theta_{r m s, w}=0: P_{r}=0.01, \mathbf{\nabla} ; P_{r}=0.1, \boldsymbol{\Lambda} . P_{r}=1$, . $P_{r}=10, \bullet . q_{w}^{\prime}=0: P_{r}=0.01, \nabla ; P_{r}=0.1, \Delta . P_{r}=1, \square . P_{r}=10, \circ . R_{\tau}=395$.

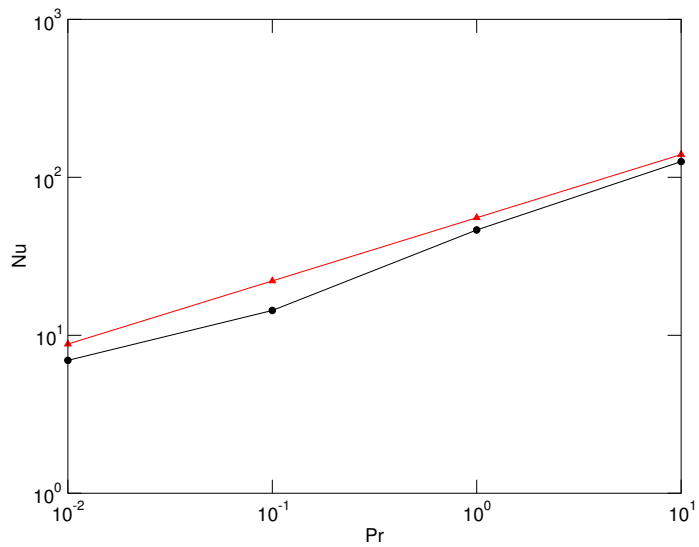

Fig. 10. Profiles of the Nusselt number versus the Prandtl number. $q_{w}^{\prime}=0$ : DNS : $\bullet$. Dittus-Boelter equation : $\mathbf{\Delta} \cdot R_{\tau}=395$.

same, the Nusselt number is therefore not affected by the scalar fluctuations at the wall. This being understood, only the profile associated with the boundary condition $q_{w}^{\prime}=0$ is plotted for consistency. In addition, the straight line corresponding to the Dittus-Boelter equation given by $N u=0.023 R e^{0.8} P_{r}^{0.4}$ originally developed for circular pipes is also indicated for comparison purpose. At a first sight, it is found that the dependence of the Nusselt number as a function of the Prandtl number is quasi-linear in logarithmic scales with the slope 0.4, even if some deviations are however visible at small Prandtl numbers. 
5 Turbulent channel flow heated on one wall and cooled on the other one with scalar fluctuations at the wall (Case II)

For illustration purpose, in addition to the Case I that has been studied above, we consider here the Case II that is a plane channel heated only from one wall but cooled from the other one at the same rate accounting for free scalar fluctuations at the wall $\theta_{w}^{\prime} \neq 0$ with $q_{w}^{\prime}=0$. The motivation is to provide another case encountered in industrial applications.

\subsection{Statistics of passive scalars}

\subsubsection{Mean scalar field}

The transformed variable $\Theta^{+}=2 \theta^{+} /\left(\theta_{w 1}^{+}-\theta_{w 2}^{+}\right)$is chosen because of the anti-symmetric thermal configuration of this case. Figure 11 shows the mean scalar profiles $\Theta^{+}$versus the dimensionless wall coordinate distance associated with several Prandtl numbers. As expected, the mean scalar profiles are perfectly anti-symmetric about the channel centerline. The boundary conditions which are imposed on the scalar variable cause the scalar gradient to be non zero in the center. As the Prandtl number increases from 0.01 to 10 , the mean profiles get steeper. This fact is resulting from the effects of the thermal diffusivity at small Prandtl number and from the momentum diffusivity at large Prandtl number. Obviously, the thermal boundary layer in the lower heated wall is identical as the one in the upper cooled wall for reasons of symmetry.

\subsubsection{Root mean square of the scalar variance}

Figure 12 displays the profiles of the rms fluctuations of the scalar fields versus the wall unit distance. As for the Case I, the intensity of the scalar variance increases with the increase of the Prandtl number. Moreover, the rms fluctuation intensities are relatively high in the immediate vicinity of the wall due to the boundary condition and even appreciable in the central region of the channel. The rms profiles are significantly different from those of Case I because of the scalar variable $\theta$ that is anti-symmetric in Case II while symmetric in Case I.

\subsubsection{Turbulent Prandtl number}

Figure 13 shows the profile of the turbulent Prandtl number for the Prandtl numbers $P_{r}=0.01,0.1,1$ and 10 versus the wall unit distance. As observed in Case I, the turbulent Prandtl number is almost constant for each Prandtl number except however at $P_{r}=0.01$. Moreover, the maximum value reached at $P_{r}=0.01$ is lower than the one obtained in Case I. Although the thermal configuration pertaining to Cases I and II is quite different from 


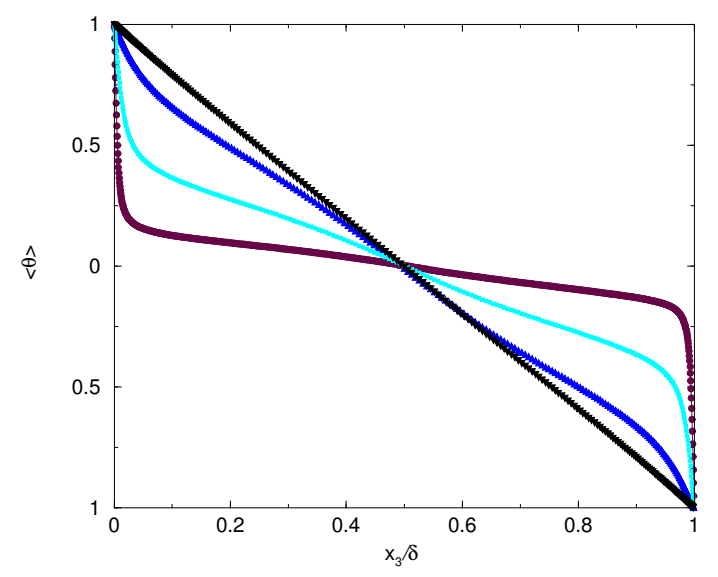

Fig. 11. Mean scalar field at different Prandtl numbers versus the wall coordinate. $P_{r}=0.01, \mathbf{\nabla} ; P_{r}=0.1, \mathbf{\Lambda} . P_{r}=1, \square . P_{r}=10$, •. $R_{\tau}=395$.

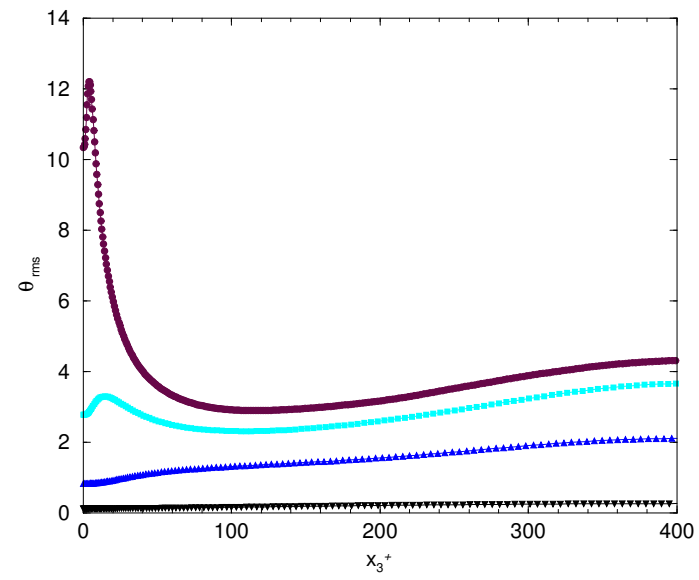

Fig. 12. Root mean square of the scalar variance versus the wall unit distance. $P_{r}=0.01, \mathbf{\nabla} ; P_{r}=0.1, \mathbf{\Delta} . P_{r}=1, \square . P_{r}=10, \bullet$. $R_{\tau}=395$.

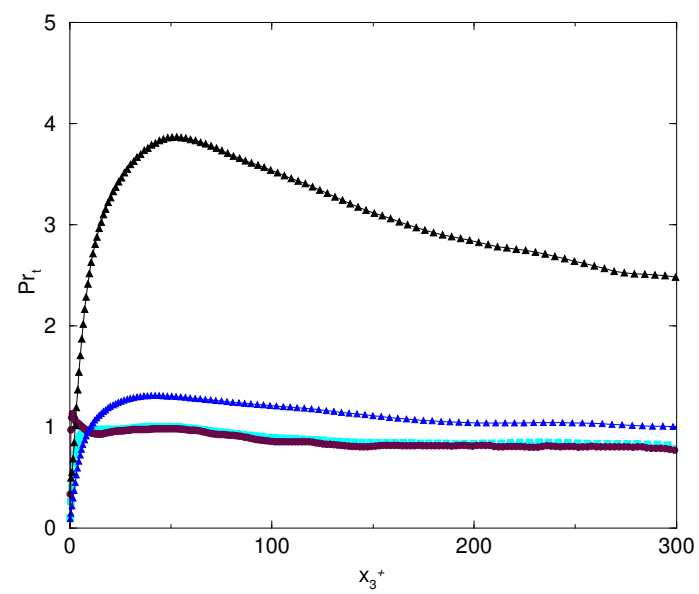

Fig. 13. Profiles of the turbulent Prandtl number versus the wall unit distance. $P_{r}=0.01, \mathbf{\nabla} ; P_{r}=0.1, \boldsymbol{\Lambda} . P_{r}=1, \square$. $P_{r}=10, \bullet$. $R_{\tau}=395$. 


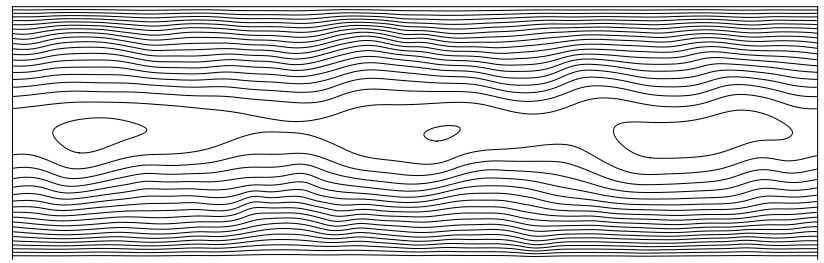

(a)

(b)

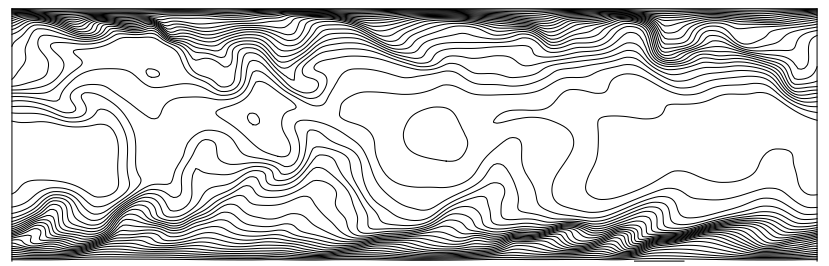

(c)

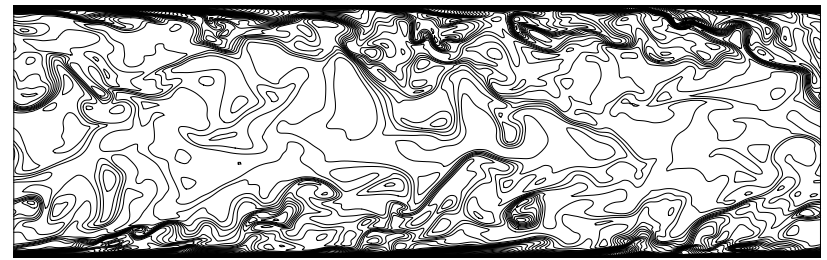

(d)

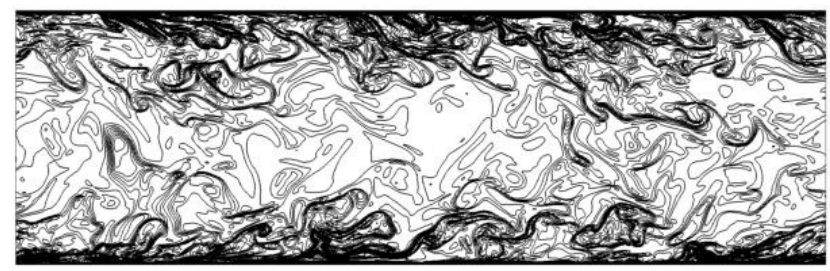

Fig. 14. Contours of the instantaneous scalar field $\Theta$ in the $\left(x_{1}, x_{3}\right)$ mid-plane. (a) $P_{r}=0.01$; (b) $P_{r}=0.1$; (c) $P_{r}=1$; (d) $P_{r}=10$; $R_{\tau}=395$.

a physical point of view, it is found in practice that the profile of the turbulent Prandtl number remains roughly unchanged at $P_{r}=0.1,1$ and 10, characterized by an asymptotic behavior around unity, but at $P_{r}=0.01$, the profile exhibits a different behavior in the near wall region.

\section{Structure of the scalar fields}

For sake of simplicity and conciseness, only the scalar field associated with the Case I accounting for zero fluctuations at the wall is investigated in this section since no significant differences with the corresponding field with wall fluctuations is visible at a first sight, at least when the Prandtl number is moderate or high. Figure 14 shows the contours plots of the instantaneous scalar field $\theta$ in the $\left(x_{1}, x_{3}\right)$ mid-plane, respectively, for the Prandtl 
numbers $P_{r}=0.01,0.1,1$ and 10. As expected, according to the evolution of the Batchelor length-scale $\eta_{\theta}$ as a function of the Prandtl number, these structures get thinner and appear less and less organized as the Prandtl numbers increase, giving rise substantially to the detachment of vortex in the normal direction to the wall. A strong activity of thermal turbulence is observed in the near wall region, characterized by the peak of rms fluctuations, as already highlighted in the preceding sections.

\section{Concluding remarks}

DNS of turbulent channel flows with passive scalar transport have been performed on different meshes at the Reynolds number $R_{\tau}=395$ and for Prandtl numbers ranging from $P_{r}=0.01,0.1,1$ to 10 with different flux conditions at the wall. The distributions of the mean scalar field, root mean square scalar fluctuations, turbulent heat flux, correlation coefficient, turbulent Prandtl number, Nusselt number, were accurately obtained from the simulation and investigated in detail. As a result of interest, it has been found that the mean scalar field $\langle\theta\rangle$ is not affected by the scalar fluctuations at the wall, but the rms scalar fluctuations is highly modified in the immediate vicinity of the wall. Surprisingly, the maximum rms intensity remains almost unchanged in the near wall region whatever the type of flux condition is applied at the wall. The turbulent heat fluxes that play a crucial role in heat transfer are found to be almost independent of the wall scalar fluctuations. However, the profiles of the correlation coefficients as well as the turbulent Prandtl number are somewhat different in the near wall region and even in the central region of the channel. Finally, the structure of the scalar fields was also examined confirming that the structures get thinner as $P_{r}$ is increasing. In addition to the Case I, the Case II corresponding to the plane channel heated only from one wall but cooled from the other one at the same rate was also performed. Further work will include the validation of turbulence models and especially hybrid RANS/LES models [20] such as the PITM developed by Chaouat and Schiestel $[26,27]$ in presence of passive scalar transport fields that is well suited for the simulation of turbulent flows encountered in industrial applications.

\section{Acknowledgements}

The authors would like to thank Dr. Roland Schiestel for his helpful discussions on this manuscript.

\section{Nomenclature}

$c_{p}=$ specific heat at constant pressure

$h=$ heat coefficient 
$K=\sqrt{\rho c_{p} \kappa / \rho_{w} c_{p w} \kappa_{w}}=$ thermal activity ratio

$L_{i}=$ computational domain size

$N u=2 \delta q_{w} /\left[\kappa\left(\theta_{w}-\theta_{b}\right)\right]=\quad$ Nusselt number

$p=$ pressure

$P_{r}=$ Prandtl number

$P_{r t}=$ turbulent Prandtl number

$q_{w}=\rho c_{p} u_{\tau} \theta_{\tau}=$ wall heat flux

$q_{i}=$ turbulent heat flux

$R_{\tau}=u_{\tau} \delta / \nu=$ friction Reynolds number

$R_{i \theta}=$ correlation coefficient

$t=$ time

$u_{i}=$ velocity in the $i$ th direction

$u_{\tau}=$ friction velocity

$U_{b}=$ bulk velocity

$x_{i}=$ coordinate in the $i$ th direction

$x_{i}^{*}=x_{i} / \delta=$ dimensionless coordinate

$x_{i}^{+}=x_{i} u_{\tau} / v=$ dimensionless coordinate

$\delta=$ half channel width

$\varepsilon=$ dissipation-rate

$\eta=$ Kolmogorov length-scale

$\eta_{\theta}=$ Batchelor length-scale

$\kappa=$ thermal conductivity

$\sigma=\kappa /\left(\rho c_{p}\right)=\mathrm{v} / P_{r} \quad$ thermal diffusivity

$\mathrm{v}=$ kinematic viscosity

$\theta=$ passive scalar variable

$\theta_{\tau}=q_{w} /\left(\rho c_{p} u_{\tau}\right)=$ friction scalar variable

$\theta_{b}=$ bulk scalar variable

$\theta_{w}=$ wall scalar variable

$\Theta=$ scalar variable deviation

$\theta_{r m s}=\sqrt{\left(\theta^{\prime} \theta^{\prime}\right)}=$ root mean-square of the scalar fluctuations 
$\rho=$ density

\section{References}

[1] Chaouat, B., 2001. "Simulations of channel flows with effects of spanwise rotation or wall injection using a Reynolds stress model”. ASME J. Fluids Eng., 123(1), pp. 2-10.

[2] Schiestel, R., 2008. Modeling and simulation of turbulent flows. ISTE Ltd, London and J. Wiley, Hoboken.

[3] Gatski, T. B., 2009. "Second-moment and scalar flux representation in engineering and geophysical flows". Fluid Dyn. Res., 41, p. 012202.

[4] Hanjalic, K., and Launder, B. E., 2011. Modelling turbulence in engineering and the environment. Secondmoment route to closure. Cambridge University Press., Cambridge.

[5] Abe, K., and Suga, K., 2001. "Towards the development of a Reynolds-averaged algebraic turbulent scalarflux model". Int. J. Heat Fluid Flow, 22(1), pp. 19-29.

[6] Kasagi, N., Kuroda, A., and Hirata, M., 1989. "Numerical investigation of near-wall turbulent heat transfer taking into account the unsteady heat conduction in the solid wall”. ASME J. Heat Transfer, 111(2), pp. 385392.

[7] Sommer, T. P., So, R. M. C., and Zhang, H. S., 1994. "Heat transfer modeling and the assumption of zero wall temperature fluctuations". ASME J. Heat Transfer, 116(4), pp. 855-863.

[8] Tiselj, I., Bergant, R., Mavko, B., Bajsić, I., and Hetsroni, G., 2001. "DNS of turbulent heat transfer in channel flow with heat conduction in the solid wall”. ASME J. Heat Transfer, 123(5), pp. 849-857.

[9] Mosyak, A., Pogrebnyak, E., and Hetsroni, G., 2001. "Effect of constant heat flux boundary condition on wall temperature fluctuations". ASME J. Heat Transfer, 123(2), pp. 213-218.

[10] Matsubara, K., Sakurai, A., Miura, T., and Kawabata, T., 2012. "Spanwise heat transport in turbulent channel flow with prandtl numbers ranging from 0.025 to 5.0". ASME J. Heat Transfer, 134(4), p. 041701.

[11] Kim, J., and Moin, P., 1989. "Transport of passive scalars in a turbulent channel flow". In Proceedings of the 6th Symposim on Turbulence Shear Flows, Springer-Verlag, pp. 85-96.

[12] Kasagi, N., Tomita, Y., and Kuroda, A., 1992. "Direct numerical simulation of passive scalar field in a turbulent channel flow". ASME J. Heat Transfer, 114(3), pp. 598-606.

[13] Kasagi, N., and Shikazono, N., 1995. "Contribution of direct numerical simulation to understanding and modelling turbulent transport". Proc. R. Soc. Lond., A, 451, pp. 257-292.

[14] Kawamura, H., Ohsaka, K., Abe, H., and Yamamoto, K., 1998. "DNS of turbulent heat transfer in channel 
flow with low to medium-high Prandtl number fluid”. Int. J. Heat Fluid Flow, 19(5), pp. 482-491.

[15] Kawamura, H., Abe, H., and Matsuo, Y., 1999. "DNS of turbulent heat transfer in channel flow with respect to Reynolds and Prandtl number effects". Int. J. Heat Fluid Flow, 29(3), pp. 196-207.

[16] Na, Y., and Hanratty, T. J., 2000. "Limiting behavior of turbulent scalar transport close to a wall”. Int. J. Heat Mass Transfer, 43(10), pp. 1749-1758.

[17] Abe, H., Kawamura, H., and Matsuo, Y., 2004. "Surface heat-flux fluctuations in a turbulent channel flow up to $R e_{\tau}=1020$ with $P_{r}=0.025$ and 0.71,”. Int. J. Heat Fluid Flow, 25(3), pp. 404-419.

[18] Kozuka, M., Seki, Y., and Kawamura, H., 2009. "DNS of turbulent heat transfer in a channel flow with a high spatial resolution". Int. J. Heat Fluid Flow, 30(3), pp. 514-524.

[19] Lu, D. M., and Hetsroni, G., 1995. "Direct numerical simulation of a turbulent open channel flow with passive heat transfer". Int. J. Heat Mass Transfer, 38(17), pp. 3241-3251.

[20] Chaouat, B., 2017. "The state of the art of hybrid RANS/LES modeling for the simulation of turbulent flows". Flow, Turbul. Combust., 99(2), pp. 279-327.

[21] Batchelor, G. K., 1959. "Small-scale variation of convected quantities like temperature in turbulent fluid. Part 1. General discussion and the case of small conductivity". J. Fluid Mech., 5(1), pp. 113-133.

[22] Batchelor, G. K., Howells, I. D., and Townsend, A. A., 1959. "Small-scale variation of convected quantities like temperature in turbulent fluid. Part 2. The case of large conductivity". J. Fluid Mech., 5(1), pp. 134-139.

[23] Tennekes, H., and Lumley, J. L., 1972. A First Course in Turbulence. The MIT Press, Cambridge.

[24] Chaouat, B., 2011. "An efficient numerical method for RANS/LES turbulent simulations using subfilter scale stress transport equations”. Int. J. Numer. Methods Fluids, 67(10), pp. 1207-1233.

[25] Moser, R., Kim, J., and Mansour, N., 1999. "Direct numerical simulation of turbulent channel up to $R e_{\tau}=$ 590”. Phys. Fluids,, 11(4), pp. 943-945.

[26] Chaouat, B., and Schiestel, R., 2005. "A new partially integrated transport model for subgrid-scale stresses and dissipation rate for turbulent developing flows". Phys. Fluids, 17(6), p. 065106.

[27] Chaouat, B., and Schiestel, R., 2013. "Hybrid RANS-LES simulations of the turbulent flow over periodic hills at high Reynolds number using the PITM method”. Comput. Fluids, 84, pp. 279-300.

\section{List of Figures}

1 Setup of the numerical channel flow simulations with various thermal boundary conditions. Both walls are heated $(A=1)$; One wall is heated but the other one is cooled at the same rate $(A=-1) . \quad \ldots \quad 4$ 
2 Turbulence intensities $u_{i r m s}^{+}=\sqrt{\left\langle u_{i}^{\prime+} u_{i}^{\prime+}\right\rangle}$ versus the wall coordinate. Present DNS performed on the mesh $M_{1}: \Delta, \mathrm{i}=1 ; \triangleleft, \mathrm{i}=2 ; \triangleright, \mathrm{i}=3$. DNS (Moser et al. 1999), $-R_{\tau}=395 . \ldots \ldots$

3 Mean scalar field $\left\langle\theta^{+}\right\rangle$at several Prandtl numbers in logarithmic coordinate versus the wall unit distance. $\theta_{r m s, w}=0, \bullet q_{w}^{\prime}=0$, . (a) $P_{r}=0.01$; (b) $P_{r}=0.1$; (c) $P_{r}=1$; (d) $P_{r}=10 ; R_{\tau}=395$.

4 Root mean square of the scalar variance $\theta_{r m s}^{+}=\sqrt{\left\langle\theta^{\prime+} \theta^{\prime+}\right\rangle}$ at several Prandtl numbers versus the wall unit distance. $\theta_{r m s, w}=0$, •. $q_{w}^{\prime}=0$, 匹. (a) $P_{r}=0.01$; (b) $P_{r}=0.1$; (c) $P_{r}=1$; (d) $P_{r}=10$;

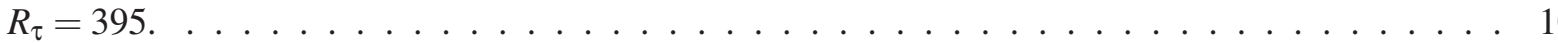

5 Normal turbulent heat flux $q_{3}=-\left\langle u_{3}^{\prime+} \theta^{\prime+}\right\rangle$ versus the wall unit distance. $\theta_{r m s, w}=0: P_{r}=0.01$, $\mathbf{\nabla} ; P_{r}=0.1, \boldsymbol{\Delta} . P_{r}=1, \square . P_{r}=10, \bullet . q_{w}^{\prime}=0: P_{r}=0.01, \nabla ; P_{r}=0.1, \Delta . P_{r}=1, \square . P_{r}=10, \circ$. $R_{\tau}=395$

6 Streamwise turbulent heat flux $\left|q_{1}\right|=\left\langle u_{1}^{\prime+} \theta^{\prime+}\right\rangle$ versus the wall unit distance. $\theta_{r m s, w}=0: P_{r}=0.01$, $\boldsymbol{\nabla} ; P_{r}=0.1, \boldsymbol{\Delta} . P_{r}=1, \square . P_{r}=10, \bullet . q_{w}^{\prime}=0: P_{r}=0.01, \nabla ; P_{r}=0.1, \Delta . P_{r}=1, \square . P_{r}=10, \circ$. $R_{\tau}=395$.

7 Correlation coefficient of the wall-normal turbulent heat flux $R_{3 \theta}$ versus the wall unit distance. $\theta_{r m s, w}=0: P_{r}=0.01, \mathbf{\nabla} ; P_{r}=0.1, \boldsymbol{\Delta} . P_{r}=1, \square . P_{r}=10, \bullet . q_{w}^{\prime}=0: P_{r}=0.01, \nabla ; P_{r}=0.1, \Delta$. $P_{r}=1, \square . P_{r}=10$, o. $R_{\tau}=395$.

8 Correlation coefficient of the streamwise turbulent heat flux $R_{1 \theta}$ versus the wall unit distance. $\theta_{r m s, w}=0: P_{r}=0.01, \mathbf{\nabla} ; P_{r}=0.1, \boldsymbol{\Delta} . P_{r}=1, \square . P_{r}=10, \bullet . q_{w}^{\prime}=0: P_{r}=0.01, \nabla ; P_{r}=0.1, \Delta$. $P_{r}=1, \square . P_{r}=10$, o. $R_{\tau}=395$.

9 Profiles of the turbulent Prandtl number versus the wall unit distance. $\theta_{r m s, w}=0: P_{r}=0.01, \boldsymbol{\nabla}$; $P_{r}=0.1, \boldsymbol{\Delta} . P_{r}=1, \square . P_{r}=10, \bullet . q_{w}^{\prime}=0: P_{r}=0.01, \nabla ; P_{r}=0.1, \Delta . P_{r}=1, \square . P_{r}=10, \circ$. $R_{\tau}=395$

10 Profiles of the Nusselt number versus the Prandtl number. $q_{w}^{\prime}=0$ : DNS : $\bullet$. Dittus-Boelter equation $: \mathbf{\Delta} \cdot R_{\tau}=395$.

11 Mean scalar field at different Prandtl numbers versus the wall coordinate. $P_{r}=0.01, \mathbf{\nabla} ; P_{r}=0.1$,

ム. $P_{r}=1, \square . P_{r}=10, \bullet . R_{\tau}=395$

12 Root mean square of the scalar variance versus the wall unit distance. $P_{r}=0.01, \mathbf{\nabla} ; P_{r}=0.1, \Delta$ $P_{r}=1, \square . P_{r}=10, \bullet . R_{\tau}=395$

13 Profiles of the turbulent Prandtl number versus the wall unit distance. $P_{r}=0.01, \mathbf{\nabla} ; P_{r}=0.1$, $P_{r}=1, \square . P_{r}=10, \bullet R_{\tau}=395$ 
14 Contours of the instantaneous scalar field $\Theta$ in the $\left(x_{1}, x_{3}\right)$ mid-plane. (a) $P_{r}=0.01$; (b) $P_{r}=0.1$;

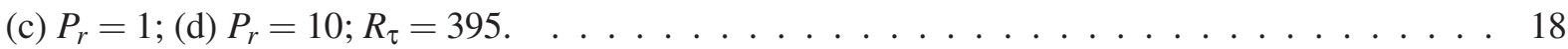

